\title{
Prevalence and association of female weight status and dietary habits with sociodemographic factors: a cross-sectional study in Saudi Arabia
}

\author{
Atika Khalaf ${ }^{1, *}$, Albert Westergren ${ }^{1}$, Vanja Berggren ${ }^{2}$, Örjan Ekblom ${ }^{3}$ \\ and Hazzaa M Al-Hazzaa ${ }^{4}$ \\ 'The PRO-CARE Group, School of Health and Society, Kristianstad University, Elmetorpsvägen 15, SE-291 88 \\ Kristianstad, Sweden: ${ }^{2}$ Department of Health Sciences, Medical Faculty, Lund University, Lund, Sweden: ${ }^{3}$ Åstrand \\ Laboratory of Work Physiology, The Swedish School of Sport and Health Sciences, Stockholm, Sweden: ${ }^{4}$ Paediatric \\ Exercise Physiology Research Laboratory, College of Education and Obesity Research, King Saud University, Riyadh, \\ Saudi Arabia
}

Submitted 17 January 2014: Final revision received 12 June 2014: Accepted 10 July 2014: First published online 4 September 2014

\begin{abstract}
Objective: Research about the prevalence of underweight and overweight/obesity in the Saudi Arabian female population is limited. The aim of the present study was to examine the dietary habits and the prevalence of underweight and overweight/obesity and associated factors among female university students.

Design: A cross-sectional study.

Setting: A university centre for female students in south-western Saudi Arabia.

Subjects: The study involved 663 randomly selected female university students who self-reported their physical activities, nutritional habits and socio-economic factors. Multiple linear and logistic regression analyses were used to identify factors associated with the students' BMI, dietary variables, underweight and overweight/obesity.

Results: The majority of the university females were normal weight (56.9\%), but a high prevalence of underweight $(19.2 \%)$ and overweight/obesity $(23.8 \%)$ occurred. Social factors significantly associated with BMI were the presence of obese parents and siblings as well as physical activity levels, marital status, number of sisters, father's level of education and more frequent intake of French fries/potato chips (>3 times/week). Several variables were found to correlate with dietary habits, underweight and overweight/obesity. Of special interest is the association between the number of siblings and the participants' BMI and dietary intake in both negative and positive ways.

Conclusions: The findings of this research have implications for health promotion and prevention of malnutrition among college-aged females. Health-care providers and policy makers need to involve the whole family when promoting females' physical activity. The study serves as an evidence-based background for planning and implementation of interventions targeting improvement of highly educated populations' nutritional habits.
\end{abstract}

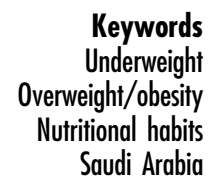

Both underweight and overweight/obesity represent a worldwide public health challenge ${ }^{(1)}$. The prevalence of obesity is increasing worldwide at an alarming rate in both developing and developed countries ${ }^{(2)}$. Obesity was estimated to be the fifth leading cause of mortality at the global level ${ }^{(3)}$. It is well recognized that obesity is associated with several chronic diseases, including CVD, diabetes mellitus, osteoporosis, osteoarthritis, hypertension and depressive disorders ${ }^{(4-7)}$.
In Saudi Arabia (KSA), obesity is a common health problem among all age groups ${ }^{(8,9)}$ and is even more common among adult females than males ${ }^{(10-12)}$. A population-based study among school-aged children and adolescents showed that the prevalence of overweight and obesity including severe obesity was $23.1 \%$ and $11.3 \%$, respectively ${ }^{(8)}$. However, data are still limited on the prevalence of underweight and overweight/obesity and associated factors among Saudi college students. 
The lifestyle habits of the Saudi female population relative to nutrition and physical activity are a bit different from those of many other countries ${ }^{(13)}$. Several genderrelated factors may contribute to the high prevalence of overweight/obesity among women in KSA. The majority of KSA women are not employed ${ }^{(14)}$ which could due in part to the higher number of female graduates but fewer job opportunities available after graduation ${ }^{(15)}$. The high prevalence of unemployment leads to increased time spent watching television and eating snacks ${ }^{(12)}$, habits known to be common during leisure time. Some in KSA might even consider overweight/obesity a sign of affluence $^{(11,12,16)}$. Another leading reason for the development of either underweight or overweight/obesity among Saudi women might be that the traditional, long, comfortable and wide clothes worn by women prevent them from noticing the gradual changes in weight ${ }^{(11,12,16)}$. An additional lifestyle habit that differs in the KSA female population is physical activity. While women in the KSA have traditionally engaged in moderately intensive physical activity through housekeeping tasks ${ }^{(17)}$, their reported prevalence of moderate and vigorous physical activity (2\%) is among the lowest in the world ${ }^{(18)}$.

With regard to university students, studies conducted among males showed that the most common eating habits encountered were eating with family, having two meals per day including breakfast, together with frequent snacks and fried foods ${ }^{(19)}$. Most students did not consume vegetables and fruits, except dates, frequently ${ }^{(19)}$. Another recent study on Saudi Arabian children and young adults aged 10-19 years has reported a positive, significant correlation between sugar-sweetened beverage consumption and poor dietary habits ${ }^{(20)}$. Moreover, research studies and reviews indicate that skipping breakfast is widespread among adolescents in the USA and Europe ${ }^{(21)}$, as well as in many Arab countries ${ }^{(14,22,23)}$. Among university female students aged 22-24 years in Riyadh, the participants skipped breakfast in $41.2 \%$ of cases, and $98.9 \%$ reported snacking ${ }^{(24)}$. That study further reported that $7.6 \%$ of the 799 participating females were underweight, while $47.9 \%$ of them were overweight/obese ${ }^{(24)}$.

There is a continuous increase in overweight/obesity in developing countries, although the prevalence of underweight is still high ${ }^{(25)}$ and is between $19 \%$ and $40 \%$ in countries such as India, Pakistan, Madagascar, Thailand and Vietnam ${ }^{(26)}$. Mendez et $a l^{(27)}$ reported that underweight remains a concern especially among women living in rural areas of the least developed countries. There are few studies on the trends in underweight and overweight/ obesity status of women in developing countries, and thus it is not known whether similar patterns have existed in the past or if these are modern occurrences ${ }^{(27)}$. In the KSA, research on underweight and undernutrition is still limited with regard to adults and to females in particular.

The health consequences related to underweight can be devastating for a society. Such health consequences could include increased mortality in response to primary viral infections because of an inability to meet the energy demands associated with the immune response to such infections ${ }^{(28)}$. However, over the past two decades there has been a documented increase in the influence of the media on weight-loss attempts, especially among women, in order to achieve the 'Western image' of an ideal body shape and weight ${ }^{(29-31)}$. The results of such weight-loss activities might lead to the development of undernutrition and underweight ${ }^{(32)}$, and researchers have found that negative attitudes towards obesity and socio-cultural preferences for thinness can even induce persons who are already underweight to attempt weight control ${ }^{(33)}$. Although underweight and its underlying factors in relation to women have not been studied in depth in the KSA, women with higher educational levels in the KSA were found to be more likely to favour slimness as an ideal body shape ${ }^{(34)}$. In addition, studies on the occurrence of underweight among KSA society as a whole and among women in particular are scarce. We attempted to fill a gap by exploring the patterns of a healthy female population's nutritional habits. Therefore, the present study aims to examine the dietary habits and the prevalence of underweight and overweight/ obesity and associated factors among female university students in south-western KSA.

\section{Methods}

\section{Design and participants}

The study was based on a cross-sectional design. The participants were college-aged females from a university centre for female studies in south-western KSA. To reach a statistical power of $80 \%$ based on a $95 \%$ confidence level, an average standard deviation of fat percentage as 7.5 (from a previous study conducted on college male students in Riyadh) ${ }^{(35)}$ and a total population of 1681 females, the sample required was about 600 students. In case some students declined participation, we included more students to reach the target sample. The sample was selected using a multistage stratified random selection procedure where 663 females were drawn equally from all four levels (freshwoman, sophomore, junior and senior levels) of university students ${ }^{(36)}$. Three of the participants were then removed during the data analysis because they were pregnant at the point of data collection. The Ethical Committee at King Khalid University, Abha, KSA (7/1078) approved the study and a written informed consent was obtained from each participant.

\section{Assessment of lifestyle habits and sociodemographic characteristics}

The protocol used in the study was a self-reported questionnaire including measures of socio-economic, environmental and cultural factors, along with physical activities, sedentary behaviours and dietary habits. The physical 
activity part of the questionnaire had been validated previously on Arab youth 15-25 years of age ${ }^{(37,38)}$ with fair and significant validity coefficients $(r=0.369, P=0.001)$.

The questionnaire included ten specific questions designed to determine the frequency of certain dietary habits of adolescents. The questions included how many times during a typical week the participants consumed breakfast, sugar-sweetened drinks including soft beverages, vegetables (cooked and uncooked), fruit, milk and dairy products, doughnuts and cakes, candy and chocolate, energy drinks and fast foods. The fast foods included examples from both Western and Arabic choices, such as shawarma (grilled meat or chicken in pita bread with salad). These questions covered healthy and unhealthy dietary habits. The students had a choice of answers, ranging from intake of 0 to a maximum of $7 \mathrm{~d}$ /week (every day). For the dietary cut-off points, we calculated the proportions of students who had a 'healthy' intake of breakfast, fruit, vegetables and milk ( $\geq 5 \mathrm{~d}$ /week) and those who had intake of the 'unhealthy' dietary choices on $<3$ d/week ${ }^{(38)}$.

Physical activity was translated into units of metabolic equivalents of task (MET), based on the compendium of physical activity ${ }^{(39)}$, and total activity energy expenditure was expressed as MET-minutes per week (MET-min/week) achieved by multiplying the intensity of the different activities (in MET) by the time spent on the activity (in min/week). For the activity levels, using cut-off points that were based on tertiles of total activity energy expenditure, persons were considered as low active when they achieved $\leq 611.56 \mathrm{MET}-\mathrm{min} /$ week, moderately active with 611.57-1389.63 MET-min/week, and high active when they achieved $\geq 1389.63 \mathrm{MET}$-min/week ${ }^{(36)}$.

In addition, anthropometric measurements were obtained including body weight (to the nearest $0.1 \mathrm{~kg}$ ), body height (to the nearest $1 \mathrm{~cm}$ ) and waist circumference (to the nearest $1 \mathrm{~cm}$ ), using a calibrated medical scale (Detecto 438, Central Carolina Scale, Sanford, NC, USA), a stadiometer (Detecto 438) and an non-stretchable measuring tape, respectively. BMI was calculated as weight in kilograms divided by the square of height in metres. BMI classifications were based on WHO cut-offs: underweight $\left(\mathrm{BMI} \leq 18.49 \mathrm{~kg} / \mathrm{m}^{2}\right)$, normal weight $\left(\mathrm{BMI}=18.50-24.99 \mathrm{~kg} / \mathrm{m}^{2}\right)$ and overweight/ obese $\left(\mathrm{BMI} \geq 25 \cdot 00 \mathrm{~kg} / \mathrm{m}^{2}\right)^{(40)}$. All socio-economic and environment-related background information was selfreported. For example, the participants subjectively assessed the distances between their residence and parks, malls and supermarkets. The students also estimated their parents' weight status subjectively.

\section{Statistical analysis}

Means, standard deviations and percentages were used for descriptive analysis. BMI classifications were used for comparisons between the groups and their association with predictor variables. Further, for each predictor variable, a reference category for further statistical analysis was created. The ordinal independent variables were analysed using the Kruskal-Wallis test (three-group comparisons) in the first step and, if significant, the MannWhitney $U$ test (two-group comparisons) in the second step. The variables with a continuous nature such as age and screen time were analysed by the parametric one-way ANOVA. Dichotomized variables were analysed using the $\chi^{2}$ test $^{(41)}$. All statistical analyses were run using the statistical software package IBM Statistics SPSS version 20.

The response quantitative variable of BMI was used as dependent variable in the multiple linear regression analyses ${ }^{(41)}$. Dummy variables (dichotomized variables) were created from the independent variables on the ordinal level and were then entered into the linear regression analysis model. Independent variables with fewer than five initial observations were not included in the analysis. In the first model for multiple linear regression (backwards method), the following predictor variables were entered: age (continuous), dietary habits (dummy variables created as shown below), economic factors (dummy variables), social and behavioural factors (dummy variables) and environmental factors (dummy variables). In the dietary habits, 'healthy' was the reference group and was compared with 'less healthy' and 'unhealthy'. The cut-offs for the 'healthy' intakes of breakfast, vegetables, fruits and milk/dairy products were $\geq 5$ times/week and for 'unhealthy' intakes, 0-4 times/week. To the contrary, for sugar-sweetened drinks, fast foods, French fries/potato chips, sweets/chocolates, cake/doughnuts/biscuits and energy drinks the cut-offs for 'healthy' and 'unhealthy' intakes were 0-2 times/week and $\geq 3$ times/week, respectively. The economic factors used in the analysis comprised parents' occupations, household monthly income and the number of cars in the household. The social and behavioural factors were marital status; presence of obese siblings and parents; father's and mother's level of education; and number of sisters; as well as activity levels and total screen time (television + computer) in hours per week. Environmental factors used in the analysis were proximity to malls and to parks. The variables that were significant in the first step were entered into a new multiple linear regression model (enter method). The probability of $F$-to-enter was set to 0.05 and $F$-to-remove was $0 \cdot 10$.

For the dietary variables and associated factors, a logistic regression analysis (backwards conditional method) was chosen $^{(41)}$. Included variables were age, marital status, father's level of education, mother's level of education, presence of obese parents, presence of obese siblings, number of brothers, number of sisters, parents' occupational status, household's monthly income measured in SAR (Saudi Arabian riyal; 1 SAR = \$US 3.75), number of cars, proximity to supermarkets, proximity to malls, activity levels and BMI. For the dependent variables of overweight/ obesity and underweight, the independent continuous variable of BMI was excluded. The probability of $F$-to-enter was set to 0.05 and $F$-to-remove to $0 \cdot 10$. 
Variables with a very low initial number were merged; for example, in father's and mother's level of education, the PhD degree or higher was merged with the category of bachelor's degree or higher. Another variable with low initial number and merged categories was the number of brothers, where the categories of no brothers and one brother were merged. Some categories in other variables were eliminated, like the category of not having any car in the variable of number of cars in the household. Further, $\alpha$ was set to $5 \%$ for statistical significance and a Bonferroni correction was carried out in the subgroup analyses. Statistical indication, on the other hand, was identified when $\alpha$ was more than $5 \%$ but less than $10 \%$.

\section{Results}

The total participating population had a mean age of $20 \cdot 4$ (SD 1.5) years. The underweight group was significantly $(P=0.01)$ younger than the normal weight and the overweight/obese groups. The prevalence of underweight, normal weight and overweight/obesity was $19.2 \%, 56.9 \%$ and $23.8 \%$, respectively (Table 1 ). As expected, BMI differed significantly among the three groups $(P<0 \cdot 001)$. The mean waist circumference also differed significantly $(P<0.001)$ between the groups: $62.5 \mathrm{~cm}$ in the underweight group, $69.5 \mathrm{~cm}$ in the normal weight group and $81.6 \mathrm{~cm}$ in the overweight/obese group (Table 1). Similarly, waist-to-height ratio $(\times 100)$ differed significantly $(P<0.001)$ between the groups: $39 \cdot 2,44.2$ and 52.2 in the underweight, normal weight and overweight/obese groups, respectively.

The proportion $(45.7 \%)$ of underweight females who were classified as low active was significantly $(P=0.013)$ higher than that found for normal weight $(31.6 \%)$ or overweight/obese females (27.4\%). Also, underweight females were the least highly active $(27 \cdot 6 \%)$ of all the three groups. There were also significant differences between the groups regarding the number of sisters, number of obese siblings and presence of obese parents. Further characteristics of the studied population with regard to economic factors and social factors are described in Table 1.

In relation to healthy dietary habits, significant differences $(P=0.001)$ were found between the groups with regard to their weekly intake of breakfast: $36 \cdot 2 \%, 51.7 \%$ and $37.6 \%$ for underweight, normal weight and overweight/obese participants, respectively. Similarly, the groups differed significantly $(P=0.035)$ in their consumption of French fries/ potato chips: $34.6 \%, 32.4 \%$ and $22.3 \%$ for overweight, normal weight and overweight/obese groups, respectively (Table 1).

\section{Determinants for BMI, dietary habits, overweight/ obesity and underweight}

The multiple linear regression model showed that $14 \%$ of the variance in BMI could be accounted for by all predictors.
Significantly associated factors were the participants' levels of activity, their marital status, presence of obese parents and siblings, the father's level of education and the intake of French fries/potato chips (Table 2). The lower activity levels had a negative effect on BMI; to be low active meant on average $0.90 \mathrm{~kg} / \mathrm{m}^{2}$ higher BMI compared with a high active person. On the other hand, marital status - namely, to be married - was shown to be positively associated with higher BMI. Married students having no children had a $1.63 \mathrm{~kg} / \mathrm{m}^{2}$ higher BMI compared with unmarried students. Similarly, married students having children had a $2.47 \mathrm{~kg} / \mathrm{m}^{2}$ higher BMI than unmarried students, on a significant level $(P=0 \cdot 010)$. Not having sisters was found to mean on average a $2.00 \mathrm{~kg} / \mathrm{m}^{2}$ higher BMI compared with having four or five sisters. Further, the presence of one obese parent affected the BMI, with $1.24 \mathrm{~kg} / \mathrm{m}^{2}$ higher BMI compared with not having an obese parent $(P=0.001)$. Likewise, the presence of two obese siblings or more was associated with BMI, which was $1.23 \mathrm{~kg} / \mathrm{m}^{2}$ (two obese siblings), $2.74 \mathrm{~kg} / \mathrm{m}^{2}$ (three obese siblings) and $4.07 \mathrm{~kg} / \mathrm{m}^{2}$ (four obese siblings or more) higher in comparison to not having an obese sibling. To have a higher-educated father was also associated with $1.03 \mathrm{~kg} / \mathrm{m}^{2}$ higher BMI compared with a primary or less-educated father $(P=0.032)$. Among the nutritional habits, the unhealthy intake of French fries/potato chips was found to mean $0.99 \mathrm{~kg} / \mathrm{m}^{2}$ higher BMI in comparison to healthy intake of French fries/potato chips (Table 2).

Table 3 presents the significant and statistically indicated results from the logistic regression models concerning the determinants for 'healthy' dietary habits. Breakfast intake was positively influenced by moderate activity level $(\mathrm{OR}=1.56 v$. high activity level) and low household monthly income of 3000 SAR or less (OR $=4 \cdot 16 v$. monthly income of 5001-10 000 SAR). The intake of vegetables was associated with parents' occupational status, with a lower intake of vegetables if only the mother works (OR $=0.18 v$. if both parents work); the presence of obese siblings, with an increased intake if four siblings or more are obese (OR $=3.89 v$. not having any obese siblings); and household income, with decreased intake if monthly income is 10001-15000 SAR (OR $=0.65 v$. monthly income of 5001-10000 SAR). With regard to consumption of fresh fruits, there were negative associations between the intake of fruits and the increased number of sisters. On the other hand, the presence of obese siblings as well as high activity levels increased the students' intake of fruits. For the milk/dairy products, the age of the participants and their residency's proximity to supermarkets were negatively associated ( $\mathrm{OR}=0.85$ and $0 \cdot 61$, respectively).

Regarding determinants of 'unhealthy' dietary habits (Table 4), the intake of sugar-sweetened drinks was significantly associated with the participants' BMI $(\mathrm{OR}=0.96)$. Further, fast-food consumption was negatively associated with the students' age $(\mathrm{OR}=0 \cdot 80)$, low activity level $(\mathrm{OR}=0.66 v$. high activity level) and number of cars in the 
Table 1 Characteristics, social, behavioural, economic and environmental factors in relation to the WHO classification of BMI among 663 randomly selected female university students, south-western Saudi Arabia

\begin{tabular}{|c|c|c|c|c|c|c|c|c|c|}
\hline & \multicolumn{6}{|c|}{ BMI classification } & \multirow[b]{3}{*}{$P$ value } & \multirow{2}{*}{\multicolumn{2}{|c|}{ Total $(n 660)$}} \\
\hline & \multicolumn{2}{|c|}{$\begin{array}{l}\text { Underweight }(n \\
127)\end{array}$} & \multicolumn{2}{|c|}{$\begin{array}{l}\text { Normal weight } \\
\quad(n 376)\end{array}$} & \multicolumn{2}{|c|}{$\begin{array}{l}\text { Overweight/ } \\
\text { obese }(n 157)\end{array}$} & & & \\
\hline & $\begin{array}{c}\text { Mean or } \\
n\end{array}$ & $\begin{array}{l}\mathrm{SD} \text { or } \\
\%\end{array}$ & $\begin{array}{c}\text { Mean or } \\
n\end{array}$ & $\begin{array}{l}\text { SD or } \\
\%\end{array}$ & $\begin{array}{l}\text { Mean or } \\
n\end{array}$ & $\begin{array}{l}\text { SD or } \\
\%\end{array}$ & & $\begin{array}{l}\text { Mean or } \\
n\end{array}$ & $\begin{array}{c}\text { SD or } \\
\%\end{array}$ \\
\hline Age (years), mean (SD) & $20 \cdot 0$ & 1.4 & $20 \cdot 5$ & 1.5 & 20.5 & 1.5 & $0.010 \dagger, \ddagger$ & $20 \cdot 4$ & 1.5 \\
\hline & \multicolumn{2}{|c|}{$18-24$} & \multicolumn{2}{|c|}{$18-25$} & \multicolumn{2}{|c|}{$18-24$} & & \multicolumn{2}{|c|}{$18-25$} \\
\hline BMI $\left(\mathrm{kg} / \mathrm{m}^{2}\right)$ & $17 \cdot 3$ & 0.9 & $21 \cdot 7$ & 1.8 & 28.5 & $2 \cdot 9$ & $<0.001 \dagger, \ddagger, \S$ & $22 \cdot 7$ & 4.2 \\
\hline Waist circumference (cm) & 62.6 & $5 \cdot 9$ & 69.5 & 6.8 & 81.6 & $8 \cdot 7$ & $<0.001 \dagger, \ddagger, \S$ & 71.4 & $9 \cdot 6$ \\
\hline Waist-to-height ratio $(\times 100)$ & 39.2 & 4.9 & 44.2 & 4.2 & $52 \cdot 2$ & 5.5 & $<0.001 \dagger, \ddagger, \S$ & 45.4 & $6 \cdot 4$ \\
\hline \multicolumn{10}{|l|}{ Parents' occupation, $n(\%)$} \\
\hline Only father works & 84 & $66 \cdot 1$ & 260 & 69.5 & 97 & $61 \cdot 8$ & & 441 & $67 \cdot 0$ \\
\hline Only mother works & 3 & $2 \cdot 4$ & 5 & 1.3 & 6 & 3.8 & & 14 & $2 \cdot 1$ \\
\hline Both parents work* & 19 & $15 \cdot 0$ & 57 & $15 \cdot 2$ & 36 & $22 \cdot 9$ & & 112 & $17 \cdot 0$ \\
\hline None of them work & 21 & $16 \cdot 5$ & 52 & 13.9 & 18 & 11.5 & & 91 & 13.8 \\
\hline \multicolumn{7}{|l|}{ Household monthly income, $n(\%)$} & 0.991 & & \\
\hline 3000 SAR or less & 10 & $7 \cdot 9$ & 17 & 4.5 & 12 & $7 \cdot 6$ & & 39 & 5.9 \\
\hline $3001-5000$ SAR & 17 & $13 \cdot 4$ & 60 & $16 \cdot 0$ & 22 & $14 \cdot 0$ & & 99 & $15 \cdot 0$ \\
\hline 5001-10000 SAR ${ }^{*}$ & 34 & $26 \cdot 8$ & 114 & 30.5 & 43 & $27 \cdot 4$ & & 191 & 29.0 \\
\hline $10000-15000$ SAR & 29 & $22 \cdot 8$ & 73 & 19.5 & 35 & $22 \cdot 3$ & & 137 & $20 \cdot 8$ \\
\hline More than 15000 SAR & 37 & $29 \cdot 1$ & 110 & $29 \cdot 4$ & 45 & 28.7 & & 192 & $29 \cdot 2$ \\
\hline Number of cars in the household, $n(\%)$ & & & & & & & 0.602 & & \\
\hline One car & 8 & $6 \cdot 3$ & 31 & 8.3 & 11 & $7 \cdot 0$ & & 50 & $7 \cdot 6$ \\
\hline Two cars* & 44 & 34.6 & 103 & $27 \cdot 6$ & 53 & 33.8 & & 200 & $30 \cdot 4$ \\
\hline Three cars or more & 75 & $59 \cdot 1$ & 239 & $64 \cdot 1$ & 93 & $59 \cdot 2$ & & 407 & 61.9 \\
\hline Marital status, $n(\%)$ & & & & & & & 0.216 & & \\
\hline Unmarried* ${ }^{*}$ & 119 & 93.7 & 353 & 93.9 & 141 & 89.9 & & 613 & $92 \cdot 9$ \\
\hline Married without children & 6 & $4 \cdot 7$ & 14 & 3.7 & 8 & $5 \cdot 1$ & & 28 & $4 \cdot 2$ \\
\hline Married with children & 2 & $1 \cdot 6$ & 9 & $2 \cdot 4$ & 8 & $5 \cdot 1$ & & 19 & $2 \cdot 9$ \\
\hline Number of sisters, $n(\%)$ & & & & & & & $0.031 \ddagger, \S$ & & \\
\hline None $^{*}$ & 3 & $2 \cdot 4$ & 12 & $3 \cdot 2$ & 9 & 5.7 & & 24 & $3 \cdot 6$ \\
\hline Only one & 11 & $8 \cdot 7$ & 26 & $6 \cdot 9$ & 18 & 11.5 & & 55 & 8.3 \\
\hline Two or three & 33 & $26 \cdot 2$ & 132 & $35 \cdot 1$ & 50 & $31 \cdot 8$ & & 215 & $32 \cdot 6$ \\
\hline Four or five & 44 & 34.9 & 122 & $32 \cdot 4$ & 55 & $35 \cdot 0$ & & 221 & 33.5 \\
\hline Six or more & 35 & $27 \cdot 8$ & 84 & $22 \cdot 3$ & 25 & $15 \cdot 9$ & & 144 & 21.9 \\
\hline Number of brothers, $n(\%)$ & & & & & & & 0.228 & & \\
\hline None $^{\star}$ & 1 & 0.8 & 6 & 1.6 & 4 & 2.5 & & 11 & 1.7 \\
\hline Only one & 10 & 7.9 & 25 & $6 \cdot 6$ & 14 & 8.9 & & 49 & $7 \cdot 4$ \\
\hline Two or three & 47 & $37 \cdot 3$ & 142 & $37 \cdot 8$ & 43 & $27 \cdot 4$ & & 232 & $35 \cdot 2$ \\
\hline Four or five & 37 & 29.4 & 119 & 31.6 & 45 & 28.7 & & 201 & 30.5 \\
\hline Six or more & 31 & 24.6 & 84 & $22 \cdot 3$ & 51 & 32.5 & & 166 & $25 \cdot 2$ \\
\hline Father's level of education, $n(\%)$ & & & & & & & 0.379 & & \\
\hline Primary or less* & 27 & $21 \cdot 3$ & 64 & $17 \cdot 0$ & 27 & $17 \cdot 2$ & & 118 & 17.9 \\
\hline Primary higher & 23 & $18 \cdot 1$ & 77 & $20 \cdot 5$ & 26 & $16 \cdot 6$ & & 126 & $19 \cdot 1$ \\
\hline Secondary & 31 & 24.4 & 86 & 22.9 & 34 & $21 \cdot 7$ & & 151 & $22 \cdot 9$ \\
\hline Bachelor's or higher & 46 & $36 \cdot 2$ & 149 & 39.6 & 70 & 44.6 & & 265 & $40 \cdot 2$ \\
\hline Mother's level of education, $n(\%)$ & & & & & & & 0.364 & & \\
\hline Primary or less* & 64 & $50 \cdot 4$ & 213 & $56 \cdot 6$ & 80 & $51 \cdot 0$ & & 357 & $54 \cdot 1$ \\
\hline Primary higher & 26 & 20.5 & 64 & $7 \cdot 0$ & 27 & $17 \cdot 2$ & & 117 & $17 \cdot 7$ \\
\hline Secondary & 21 & $16 \cdot 5$ & 47 & $12 \cdot 5$ & 25 & $15 \cdot 9$ & & 93 & $14 \cdot 1$ \\
\hline Bachelor's or higher & 16 & $12 \cdot 6$ & 52 & $13 \cdot 8$ & 25 & $15 \cdot 9$ & & 93 & $14 \cdot 1$ \\
\hline Presence of obese siblings, $n(\%)$ & & & & & & & $<0.001 \ddagger$,§ & & \\
\hline No one is obese* & 74 & $58 \cdot 3$ & 191 & $50 \cdot 8$ & 49 & $31 \cdot 2$ & & 314 & $47 \cdot 6$ \\
\hline Only one & 22 & $17 \cdot 3$ & 83 & $22 \cdot 1$ & 33 & $21 \cdot 0$ & & 138 & 20.9 \\
\hline Two & 21 & $16 \cdot 5$ & 56 & 14.9 & 32 & $20 \cdot 4$ & & 109 & $16 \cdot 5$ \\
\hline Three & 5 & 3.9 & 34 & $9 \cdot 0$ & 23 & $14 \cdot 6$ & & 62 & $9 \cdot 4$ \\
\hline Four or more & 5 & 3.9 & 12 & 3.2 & 20 & $12 \cdot 7$ & & $5 \cdot 6$ & $5 \cdot 6$ \\
\hline Presence of obese parents, $n(\%)$ & & & & & & & $<0.001 \ddagger, \S$ & & \\
\hline None is obese* & 88 & $69 \cdot 8$ & 228 & $60 \cdot 6$ & 65 & 41.4 & & 381 & $57 \cdot 8$ \\
\hline One/both parents is/are obese & 38 & $30 \cdot 2$ & 148 & $39 \cdot 4$ & 92 & $58 \cdot 6$ & & 278 & $42 \cdot 2$ \\
\hline $\begin{array}{l}\text { Screen time (TV viewing and computer use) }(\mathrm{h} / \mathrm{d}) \text {, } \\
\text { mean (SD) }\end{array}$ & $5 \cdot 0$ & 3.4 & $4 \cdot 8$ & 3.3 & $4 \cdot 8$ & $3 \cdot 2$ & 0.723 & $4 \cdot 8$ & $3 \cdot 3$ \\
\hline Activity levels (MET-min/week), $n$ (\%) & & & & & & & $0.013 \dagger, \ddagger$ & & \\
\hline Low active & 58 & $45 \cdot 7$ & 119 & 31.6 & 43 & $27 \cdot 4$ & & 220 & $33 \cdot 3$ \\
\hline Moderately active & 34 & $26 \cdot 8$ & 130 & $34 \cdot 6$ & 56 & $35 \cdot 7$ & & 220 & $33 \cdot 3$ \\
\hline High active $^{*}$ & 35 & $27 \cdot 6$ & 127 & 33.8 & 58 & $36 \cdot 9$ & & 220 & $33 \cdot 3$ \\
\hline
\end{tabular}




\begin{tabular}{|c|c|c|c|c|c|c|c|c|c|}
\hline & \multicolumn{6}{|c|}{ BMI classification } & \multirow[b]{3}{*}{$P$ value } & \multirow{2}{*}{\multicolumn{2}{|c|}{ Total $(n 660)$}} \\
\hline & \multicolumn{2}{|c|}{$\begin{array}{c}\text { Underweight ( } n \\
127)\end{array}$} & \multicolumn{2}{|c|}{$\begin{array}{l}\text { Normal weight } \\
\quad(n 376)\end{array}$} & \multicolumn{2}{|c|}{$\begin{array}{l}\text { Overweight/ } \\
\text { obese ( } n \text { 157) }\end{array}$} & & & \\
\hline & $\begin{array}{c}\text { Mean or } \\
n\end{array}$ & $\begin{array}{l}\text { SD or } \\
\%\end{array}$ & $\begin{array}{c}\text { Mean or } \\
n\end{array}$ & $\begin{array}{c}\text { SD or } \\
\%\end{array}$ & $\begin{array}{c}\text { Mean or } \\
n\end{array}$ & $\begin{array}{l}\text { SD or } \\
\%\end{array}$ & & $\begin{array}{c}\text { Mean or } \\
n\end{array}$ & $\begin{array}{c}\text { SD or } \\
\%\end{array}$ \\
\hline Proximity to supermarkets, $n(\%)$ & & & & & & & 0.903 & & \\
\hline Very close* & 28 & $22 \cdot 0$ & 79 & $21 \cdot 0$ & 35 & $22 \cdot 3$ & & 142 & 21.5 \\
\hline Kind of close & 77 & $60 \cdot 6$ & 230 & $61 \cdot 2$ & 89 & $56 \cdot 7$ & & 396 & $60 \cdot 0$ \\
\hline Far from house & 22 & $17 \cdot 3$ & 67 & $17 \cdot 8$ & 33 & $221 \cdot 0$ & & 122 & 18.5 \\
\hline Proximity to malls, $n(\%)$ & & & & & & & 0.740 & & \\
\hline Very close* & 6 & $4 \cdot 7$ & 18 & $4 \cdot 8$ & 4 & 2.5 & & 28 & 4.2 \\
\hline Kind of close & 51 & $40 \cdot 2$ & 135 & 35.9 & 63 & $40 \cdot 1$ & & 249 & 37.7 \\
\hline Far from house & 70 & $55 \cdot 1$ & 223 & $59 \cdot 3$ & 90 & $57 \cdot 3$ & & 383 & 58.0 \\
\hline Healthy dietary habits, $n(\%) \|$ & & & & & & & & & \\
\hline Breakfast ( $\geq 5 \mathrm{~d} /$ week) & 46 & $36 \cdot 2$ & 194 & $51 \cdot 7$ & 59 & 37.6 & $0.001+, \S$ & 299 & 45.4 \\
\hline Vegetables ( $\geq 5 \mathrm{~d} /$ week) & 55 & 43.3 & 181 & $48 \cdot 1$ & 78 & 49.7 & 0.534 & 314 & $47 \cdot 6$ \\
\hline Fruits ( $\geq 5 \mathrm{~d} /$ week) & 28 & $22 \cdot 0$ & 119 & 31.6 & 52 & $33 \cdot 1$ & 0.081 & 199 & $30 \cdot 2$ \\
\hline Milk/dairy products ( $\geq 5 \mathrm{~d} /$ week) & 73 & 57.5 & 211 & $56 \cdot 1$ & 90 & $57 \cdot 3$ & 0.947 & 374 & $56 \cdot 7$ \\
\hline Sugar-sweetened drinks ( $<3 \mathrm{~d} /$ week) & 71 & 55.9 & 192 & $51 \cdot 1$ & 73 & 46.5 & 0.287 & 336 & $50 \cdot 9$ \\
\hline Fast foods ( $<3 \mathrm{~d} /$ week) & 19 & $15 \cdot 0$ & 51 & 13.6 & 20 & $12 \cdot 7$ & 0.861 & 90 & 13.6 \\
\hline French fries/potato chips ( $<3 \mathrm{~d} /$ week) & 44 & 34.6 & 122 & 32.4 & 35 & $22 \cdot 3$ & $0.036 \ddagger$ & 201 & 30.5 \\
\hline Sweets/chocolates $(<3 \mathrm{~d} /$ week $)$ & 73 & $57 \cdot 5$ & 206 & $54 \cdot 8$ & 76 & $48 \cdot 4$ & 0.262 & 355 & 53.8 \\
\hline Cake/doughnuts/biscuits ( $<3 \mathrm{~d} /$ week) & 55 & 43.3 & 127 & $33 \cdot 8$ & 49 & 31.2 & 0.078 & 231 & 35.0 \\
\hline Energy drinks $(<3 \mathrm{~d} /$ week) & 122 & $96 \cdot 1$ & 364 & $96 \cdot 8$ & 152 & $96 \cdot 8$ & 0.915 & 638 & 96.7 \\
\hline
\end{tabular}

SAR, Saudi Arabian riyal; TV, television; MET, metabolic equivalents of task.

Underweight, $\mathrm{BMI} \leq 18.5 \mathrm{~kg} / \mathrm{m}^{2}$; normal weight, $\mathrm{BMI}=18.50-24.99 \mathrm{~kg} / \mathrm{m}^{2}$; overweight, $\mathrm{BMI} \geq 25.00 \mathrm{~kg} / \mathrm{m}^{2}$

Statistical comparisons: ordinal level, Kruskal-Wallis test (three-group comparisons) and Mann-Whitney $U$ test (two-group comparisons); continuous variables, one-way ANOVA; dichotomized variables, $x^{2}$ test. Statistical significance at $P=0.05$ and statistical indication at $P=0 \cdot 10$.

${ }^{*}$ Reference category for further statistical analysis.

†Significant difference between the underweight group and the normal weight group, after Bonferroni correction.

†Significant difference between the underweight group and the overweight/obese group, after Bonferroni correction.

§Significant difference between the normal weight group and the overweight/obese group, after Bonferroni correction.

IIHealthy food intake, i.e. intake $\geq 5$ times/week of breakfast, vegetables, fruits and milk/dairy products, and intake $<3$ times/week of sugar-sweetened drinks, fast foods, French fries/potato chips, sweets/chocolates, cake/doughnuts/biscuits and energy drinks. Dichotomized variables.

Table 2 Multiple linear regression analysis: variables associated with BMI among 663 randomly selected female university students, southwestern Saudi Arabia

\begin{tabular}{|c|c|c|c|c|}
\hline Variable & $\begin{array}{l}\text { Unstandardized } \\
\text { coefficient } b\end{array}$ & $\begin{array}{l}95 \% \mathrm{Cl} \text { for } \\
\text { coefficient } b\end{array}$ & $\begin{array}{l}\text { Standardized } \\
\text { coefficient } \beta\end{array}$ & $\begin{array}{c}P \\
\text { value }\end{array}$ \\
\hline Activity levels $(0=\text { others, } 1=\text { low active })^{*}$ & -0.90 & $-1.66,-0.14$ & $-0 \cdot 10$ & 0.021 \\
\hline Marital status $(0=$ others, $1=$ married no children $) \dagger$ & 1.63 & $0.04,3.23$ & 2.01 & 0.045 \\
\hline Marital status $(0=$ others, $1=$ married having children $) \dagger$ & 2.47 & $0.61,4.34$ & $2 \cdot 60$ & 0.010 \\
\hline Number of sisters $(0=$ others, $1=$ none $) \ddagger$ & 2.00 & $0.26,3.75$ & 2.25 & 0.025 \\
\hline Obese parents $(0=$ others, $1=$ mother or father $) \S$ & 1.24 & $0.54,1.93$ & 3.48 & 0.001 \\
\hline Obese siblings $(0=$ others, $1=$ two $) \|$ & 1.23 & $0.32,2 \cdot 13$ & $2 \cdot 66$ & 0.008 \\
\hline Obese siblings $(0=$ others, $1=$ three $) \|$ & $2 \cdot 74$ & $1.59,3.89$ & 4.68 & $<0.001$ \\
\hline Obese siblings $(0=$ others, $1=$ four or more $) \|$ & 4.07 & $2.65,5.49$ & $5 \cdot 63$ & $<0.001$ \\
\hline Father's level of education ( $0=$ others, $1=$ bachelor's degree $) \mathbb{\|}$ & 1.03 & $0.09,1.96$ & $2 \cdot 15$ & 0.032 \\
\hline Intake of French fries/potato chips $(0=\text { others, } 1=\text { unhealthy })^{\star \star}$ & 0.99 & $0.21,1.67$ & $2 \cdot 51$ & 0.012 \\
\hline
\end{tabular}

Adjusted $R^{2}=0.140$. Model $P<0.0001$.

${ }^{*}$ Reference category is high active.

tReference category is unmarried.

$\ddagger$ Reference category is four or five sisters.

$\S$ Reference category is none of the parents is obese.

IIReference category is none of the siblings is obese.

TReference category is primary education or less.

${ }^{* \star}$ Reference category is healthy intake ( $<3$ times/week).

household ( $\mathrm{OR}=0.45$ for one car $v$. two cars). Regarding the intake of French fries and potato chips, the correlations were with BMI $(\mathrm{OR}=0.95)$ and the presence of one obese parent $(\mathrm{OR}=1.47 v$. no obese parent). The consumption of sweets/chocolate decreased significantly with increased
BMI $(\mathrm{OR}=0.94)$ and if only the mother worked $(\mathrm{OR}=0.24$ $v$. both parents worked), and increased with increased proximity to malls $(\mathrm{OR}=3.19$ and 3.31 for kind of close to residence and far from residence, respectively, $v$. very close) and higher level of the father's education $(\mathrm{OR}=2.03$ 
Table 3 Significant determinants for healthy food habits: related odds ratio and $95 \%$ confidence intervals among 663 randomly selected female university students, south-western Saudi Arabia

\begin{tabular}{|c|c|c|c|c|}
\hline Dependent variables & Determinants & OR & $95 \% \mathrm{Cl}$ & $P$ value \\
\hline \multirow[t]{8}{*}{ Breakfast† } & Activity levels, high active* & & & 0.077 \\
\hline & Low active & 0.88 & $0.59,1.31$ & 0.528 \\
\hline & Moderately active & 1.56 & $1.05,2.32$ & 0.029 \\
\hline & Household monthly income, 5001-10000 SAR & & & 0.016 \\
\hline & 3000 SAR or less & $4 \cdot 16$ & $1.64,10.53$ & 0.003 \\
\hline & $3001-5000$ SAR & 1.11 & $0.67,1.83$ & 0.693 \\
\hline & $10000-15000$ SAR & 0.78 & $0.50,1.24$ & 0.295 \\
\hline & More than 15000 SAR & 1.01 & $0.67,1.53$ & 0.962 \\
\hline \multirow{14}{*}{ Vegetables $\ddagger$} & Parents' occupational status, only father works* & & & 0.143 \\
\hline & Only father works & 0.87 & $0.55,1.38$ & 0.556 \\
\hline & Only mother works & $0 \cdot 18$ & $0.04,0.76$ & 0.020 \\
\hline & None of them work & 0.81 & $0.42,1.57$ & 0.537 \\
\hline & Obese siblings, none is obese ${ }^{*}$ & & & 0.033 \\
\hline & Only one & 1.20 & $0.79,1.82$ & 0.399 \\
\hline & Two & 0.99 & $0.62,1.58$ & 0.969 \\
\hline & Three & 1.26 & $0.71,2 \cdot 26$ & 0.428 \\
\hline & Four or more & 3.89 & $1 \cdot 66,9 \cdot 14$ & 0.002 \\
\hline & Household monthly income, 5001-10000 SAR & & & 0.015 \\
\hline & 3000 SAR or less & 1.51 & $0.69,3.28$ & 0.303 \\
\hline & $3001-5000$ SAR & 1.49 & $0.88,2.52$ & 0.137 \\
\hline & $10000-15000$ SAR & 0.65 & $0.41,1.04$ & 0.073 \\
\hline & More than 15000 SAR & 0.68 & $0.43,1.06$ & 0.092 \\
\hline \multirow[t]{13}{*}{ Fresh fruits§ } & Number of sisters, none ${ }^{*}$ & & & 0.126 \\
\hline & Only one & 0.31 & $0.08,1.21$ & 0.092 \\
\hline & Two or three & 0.32 & $0.09,1.15$ & 0.080 \\
\hline & Four or five & 0.42 & $0.12,1.48$ & 0.176 \\
\hline & Six or more & 0.25 & $0.07,0.92$ & 0.037 \\
\hline & Obese siblings, none is obese* & & & 0.133 \\
\hline & Only one & 1.26 & $0.80,2.01$ & 0.320 \\
\hline & Two & 0.84 & $0.52,1.36$ & 0.472 \\
\hline & Three & 0.87 & $0.48,1.58$ & 0.649 \\
\hline & Four or more & 2.94 & $1.09,7.96$ & 0.034 \\
\hline & Activity levels, high active* & & & 0.013 \\
\hline & Low active & 1.45 & $0.93,2.25$ & 0.103 \\
\hline & Moderately active & 0.75 & $0.50,1.14$ & 0.177 \\
\hline \multirow{4}{*}{ Milk/dairy products॥ } & Age & 0.85 & $0.76,0.95$ & 0.003 \\
\hline & Proximity to supermarkets, very close* & & & 0.065 \\
\hline & Kind of close & 1.03 & $0.69,1.53$ & 0.895 \\
\hline & Far from residency & 0.61 & $0.36,1.03$ & 0.063 \\
\hline
\end{tabular}

SAR $=$ Saudi Arabian riyal.

Included variables: age, marital status, father's level of education, mother's level of education, presence of obese parents, presence of obese siblings, number of brothers, number of sisters, parents' occupational status, household's monthly income, number of cars, proximity to supermarkets, proximity to malls, activity levels and BMI.

${ }^{*}$ Reference category.

†Nagelkerke $R^{2}=0.043$; Hosmer and Lemeshow test, $X^{2}=14.659, P=0.066$.

†Nagelkerke $R^{2}=0.064$; Hosmer and Lemeshow test, $X^{2}=1.926, P=0.983$.

$\S$ Nagelkerke $R^{2}=0.056$; Hosmer and Lemeshow test, $X^{2}=10.800, P=0.213$.

IINagelkerke $R^{2}=0.029$; Hosmer and Lemeshow test, $X^{2}=9.840, P=0.276$.

and 2.27 for primary higher and bachelor's or higher, respectively, $v$. primary or less). Similarly, the consumption of cake/doughnuts/biscuits decreased significantly with increased BMI $(\mathrm{OR}=0 \cdot 96)$. The energy drink consumption correlated with higher level of mother's education $(\mathrm{OR}=$ $12.56,8.63$ and 24.28 for primary higher, secondary and bachelor's or higher, respectively, $v$. primary education or less) and lower household income (OR $=4.05$ for monthly income of 3000 SAR or less $v$. 5001-10 000 SAR).

The participants' age, presence of three obese siblings and presence of two obese parents increased the females' underweight, while having six or more sisters and being low physically active affected the underweight negatively (Table 5). Overweight/obesity increased significantly with an increased number of brothers $(\mathrm{OR}=2 \cdot 13$ for two or three brothers $v$. none or one), an increased of number of sisters $(\mathrm{OR}=5.55$ for six or more sisters $v$. no sister) and if none of the parents were working $(\mathrm{OR}=2.67 v$. both parents work); and decreased in relation to an increased number of obese siblings $(\mathrm{OR}=0.12$ for four or more $v$. none) and the presence of one obese parent $(\mathrm{OR}=0.47 v$. no obese parent).

\section{Discussion}

The study's main findings are: (i) underweight was almost as prevalent as overweight/obesity among female university 
Table 4 Significant determinants for unhealthy food habits: related odds ratios and $95 \%$ confidence intervals among 663 randomly selected female university students, south-western Saudi Arabia

\begin{tabular}{|c|c|c|c|c|}
\hline Dependent variables & Determinants & OR & $95 \% \mathrm{Cl}$ & $P$ value \\
\hline \multirow[t]{4}{*}{ Sugar-sweetened drinks† } & $\mathrm{BMI}$ & 0.96 & $0.92,1.00$ & 0.040 \\
\hline & Marital status, unmarried* & & & 0.081 \\
\hline & Married no children & $2 \cdot 26$ & $0.76,6.71$ & 0.143 \\
\hline & Married with children & 0.45 & $0.17,1 \cdot 17$ & 0.101 \\
\hline \multirow[t]{7}{*}{ Fast foods $\ddagger$} & Age & 0.80 & $0.71,0.90$ & $<0.001$ \\
\hline & Activity levels, high active* & & & 0.088 \\
\hline & Low active & 0.66 & $0.44,0.98$ & 0.042 \\
\hline & Moderately active & 0.71 & $0.48,1.05$ & 0.084 \\
\hline & Number of cars, two cars* & & & 0.025 \\
\hline & One car & 0.45 & $0.21,0.95$ & 0.035 \\
\hline & Three cars or more & $1 \cdot 19$ & $0.83,1.71$ & 0.349 \\
\hline \multirow{7}{*}{ French fries/potato chips§ } & BMI & 0.95 & $0.91,0.99$ & 0.007 \\
\hline & Obese parents, none is obese ${ }^{*}$ & & & 0.019 \\
\hline & One of the parents is obese & 1.47 & $1 \cdot 03,2 \cdot 11$ & 0.034 \\
\hline & Both parents are obese & 0.67 & $0.36,1.23$ & 0.193 \\
\hline & Number of cars, two cars* & & & 0.099 \\
\hline & One car & 0.69 & $0.36,1.33$ & 0.271 \\
\hline & Three cars or more & 1.27 & $0.89,1.81$ & 0.189 \\
\hline \multirow[t]{12}{*}{ Sweets/chocolates\|ll } & BMI & 0.94 & $0.90,0.98$ & 0.005 \\
\hline & Proximity to malls, very close* & & & 0.023 \\
\hline & Kind of close & $3 \cdot 19$ & $1.32,7.72$ & 0.010 \\
\hline & Far from house & $3 \cdot 31$ & $1.40,7.83$ & 0.006 \\
\hline & Parents' occupation, both parents work & & & 0.060 \\
\hline & Only father works & 0.56 & $0.30,1.04$ & 0.067 \\
\hline & Only mother works & 0.24 & $0.07,0.85$ & 0.027 \\
\hline & None of them work & 0.83 & $0.36,1.92$ & 0.667 \\
\hline & Father's level of education, primary or less ${ }^{*}$ & & & 0.019 \\
\hline & Primary higher & 2.03 & $1.07,3.84$ & 0.030 \\
\hline & Secondary & 1.32 & $0.74,2.36$ & 0.348 \\
\hline & Bachelor or higher & $2 \cdot 27$ & $1.27,4.04$ & 0.005 \\
\hline \multirow[t]{12}{*}{ Cake/doughnuts/biscuits } & $\mathrm{BMI}$ & 0.96 & $0.92,1.00$ & 0.034 \\
\hline & Number of sisters, none* & & & 0.082 \\
\hline & Only one & 0.66 & $0.24,1.83$ & 0.429 \\
\hline & Two or three & 1.29 & $0.53,3.17$ & 0.574 \\
\hline & Four or five & $1 \cdot 16$ & $0.47,2.84$ & 0.747 \\
\hline & Six or more & 1.71 & $0.67,4.34$ & 0.259 \\
\hline & Proximity to malls, very close* & & & 0.096 \\
\hline & Kind of close & $2 \cdot 28$ & $0.99,5.27$ & 0.053 \\
\hline & Far from house & 1.76 & $0.78,3.99$ & 0.173 \\
\hline & Number of cars, two cars* & & & 0.036 \\
\hline & One car & 0.56 & $0.29,1.06$ & 0.073 \\
\hline & Three cars or more & 1.22 & $0.84,1.75$ & 0.294 \\
\hline \multirow{10}{*}{ Energy drinks ${ }^{\star *}$} & Age & 0.74 & $0.52,1.06$ & 0.103 \\
\hline & Mother's level of education, primary or less* & & & 0.001 \\
\hline & Primary higher & 12.56 & $2 \cdot 88,54 \cdot 84$ & 0.001 \\
\hline & Secondary & 8.63 & $1.46,50 \cdot 85$ & 0.017 \\
\hline & Bachelor's or higher & $24 \cdot 28$ & $4.96,118.77$ & $<0.001$ \\
\hline & Household monthly income, 5001-10000 SAR & & & 0.028 \\
\hline & 3000 SAR or less & 4.05 & $0.37,43.73$ & 0.250 \\
\hline & $3001-5000$ SAR & 4.05 & $1.02,16.09$ & 0.047 \\
\hline & $10000-15000$ SAR & 0.37 & $0.06,2 \cdot 11$ & 0.260 \\
\hline & More than 15000 SAR & 0.66 & $0.18,2.43$ & 0.530 \\
\hline
\end{tabular}

SAR, Saudi Arabian riyal.

Included variables: age, marital status, father's level of education, mother's level of education, presence of obese parents, presence of obese siblings, number of brothers, number of sisters, parents' occupational status, household's monthly income, number of cars, proximity to supermarkets, proximity to malls, activity levels and BMI.

${ }^{*}$ Reference category.

†Nagelkerke $R^{2}=0.023$; Hosmer and Lemeshow test, $X^{2}=1.731, P=0.988$

¥Nagelkerke $R^{2}=0.063$; Hosmer and Lemeshow test, $X^{2}=9.383, P=0.311$

$\S$ Nagelkerke $R^{2}=0.039$; Hosmer and Lemeshow test, $X^{2}=9.488, P=0.303$

IINagelkerke $R^{2}=0.073$; Hosmer and Lemeshow test, $X^{2}=8.550, P=0.382$.

INagelkerke $R^{2}=0.055$; Hosmer and Lemeshow test, $X^{2}=8.011, P=0.432$.

${ }^{\star *}$ Nagelkerke $R^{2}=0.187$; Hosmer and Lemeshow test, $X^{2}=5.690, P=0.682$.

students; (ii) marital status, parents' level of education and social and family-related factors such as number of brothers and sisters influenced the participants' BMI and dietary intake in both negative and positive ways; (iii) healthy intake of breakfast and French fries/potato chips differed significantly between the groups of overweight, normal 
Table 5 Significant determinants for overweight/obesity and underweight: related odds ratios and $95 \%$ confidence intervals among 663 randomly selected female university students, south-western Saudi Arabia

\begin{tabular}{|c|c|c|c|c|}
\hline Dependent variables & Determinants & OR & $95 \% \mathrm{Cl}$ & $P$ value \\
\hline \multirow[t]{17}{*}{ Underweight† } & Age & $1 \cdot 28$ & $1.09,1.52$ & 0.003 \\
\hline & Number of sisters, none* & & & 0.046 \\
\hline & Only one & 0.22 & $0.03,1.95$ & 0.175 \\
\hline & Two or three & 0.26 & $0.03,2.05$ & 0.200 \\
\hline & Four or five & 1.18 & $0.02,1.41$ & 0.103 \\
\hline & Six or more & 0.12 & $0.01,0.91$ & 0.041 \\
\hline & Presence of obese siblings, none is obese* & & & 0.173 \\
\hline & Only one & 1.39 & $0.76,2.52$ & 0.282 \\
\hline & Two & 1.18 & $0.61,2.26$ & 0.627 \\
\hline & Three & 5.75 & $1.32,24.97$ & 0.020 \\
\hline & Four or more & 1.69 & $0.53,5.35$ & 0.374 \\
\hline & Presence of obese parents, none is obese ${ }^{*}$ & & & 0.077 \\
\hline & One of the parents is obese & 1.58 & $0.93,2.69$ & 0.091 \\
\hline & Both parents are obese & 3.00 & $0.87,10.33$ & 0.082 \\
\hline & Activity levels, high active* & & & 0.004 \\
\hline & Low active & 0.44 & $0.25,0.77$ & 0.004 \\
\hline & Moderately active & 0.96 & $0.52,1.75$ & 0.881 \\
\hline \multirow{21}{*}{ Overweight/obesity $\ddagger$} & Number of brothers, none or one ${ }^{*}$ & & & 0.089 \\
\hline & Two or three & $2 \cdot 13$ & $1.06,4.26$ & 0.034 \\
\hline & Four or five & 1.85 & $0.91,3.79$ & 0.090 \\
\hline & Six or more & 1.30 & $0.64,2.65$ & 0.462 \\
\hline & Number of sisters, none* & & & 0.004 \\
\hline & Only one & $1 \cdot 60$ & $0.53,4.79$ & 0.402 \\
\hline & Two or three & 3.19 & $1.20,8.51$ & 0.020 \\
\hline & Four or five & 3.52 & $1.31,9.49$ & 0.013 \\
\hline & Six or more & 5.55 & $1.94,15.85$ & 0.001 \\
\hline & Presence of obese siblings, none is obese ${ }^{*}$ & & & $<0.001$ \\
\hline & Only one & 0.63 & $0.37,1.08$ & 0.092 \\
\hline & Two & 0.45 & $0.26,0.80$ & 0.006 \\
\hline & Three & 0.33 & $0.17,0.65$ & 0.001 \\
\hline & Four or more & 0.12 & $0.05,0.29$ & $<0.001$ \\
\hline & Presence of obese parents, none is obese* & & & 0.002 \\
\hline & One of the parents is obese & 0.47 & $0.31,0.71$ & $<0.001$ \\
\hline & Both parents are obese & 0.69 & $0.34,1.42$ & 0.315 \\
\hline & Parents' occupational status, both parents work* & & & 0.033 \\
\hline & Only father works & 1.68 & $1.03,2.77$ & 0.039 \\
\hline & Only mother works & 0.81 & $0.22,3.01$ & 0.751 \\
\hline & None of them work & 2.67 & $1.28,5.57$ & 0.009 \\
\hline
\end{tabular}

SAR, Saudi Arabian riyal.

Included variables: age, marital status, father's level of education, mother's level of education, presence of obese parents, presence of obese siblings, number of brothers, number of sisters, parents' occupational status, household's monthly income, number of cars, proximity to supermarkets, proximity to malls, and activity levels.

${ }^{*}$ Reference category,

†Nagelkerke $R^{2}=0.133$; Hosmer and Lemeshow test, $X^{2}=11.956, P=0.153$.

$\ddagger$ Nagelkerke $R^{2}=0.179$; Hosmer and Lemeshow test, $X^{2}=10.022, P=0.263$.

weight and overweight/obese, while an unhealthy intake of French fries/potato chips was related to higher BMI; and (iv) there were negative associations between the participants' physical activity levels and their BMI, i.e. the more physically active the participants were, the lower BMI they had.

The finding that underweight was highly prevalent among this healthy and highly educated sample of Saudi female university students is similar to results reported previously for a nearby university population in the United Arab Emirates ${ }^{(42)}$. The similarities consist of the targeted age group (18-24 years), the gender (females) and the prevalence of underweight (20\%) compared with the overweight/obesity $(31.5 \%)$ in this university population. Contrary to the findings of the present study, Al-Rethaiaa and colleagues ${ }^{(19)}$ reported only $5 \%$ of underweight male students in a university-based population ( $n$ 357) in KSA, Qassim Province. In the current study, factors found to be significantly associated with the participants' underweight were age, number of sisters, presence of obese siblings or parents, and the physical activity levels of the participants. Several previous studies were conducted with a special focus on overweight/obesity ${ }^{(16,19,43,44)}$, whereas less is known about the prevalence of underweight in healthy populations. Because the present study shows a high level of underweight students, the phenomenon should be emphasized further and targeted in future research regarding body weight and associated factors. Such studies should also focus on whether the underweight is unintentional (due to lack of appetite, economic limitations, etc.) or if it is due to eating disorders. The results of such studies could be beneficial to health-care authorities and policy 
makers to prevent many health conditions related to underweight such as low immunity diseases, for example, tuberculosis $^{(45)}$. Infectious diseases ${ }^{(46,47)}$ and osteoporosis ${ }^{(47)}$ are other health conditions related to underweight that can be averted.

With regard to the nutritional habits of the population, it was shown that overweight/obese students had the highest intake of unhealthy foods. A recently published study conducted on male university students $(21.8 \%$ overweight and $15.7 \%$ obese) in $\mathrm{KSA}^{(19)}$ indicated that the students' most common eating habits were eating with family, having two meals per day including breakfast, combined with frequent snacks and fried foods, which is consistent with the results of current study. Further, vegetables and fruits were not frequently consumed by most students ${ }^{(19)}$, a result similar to our study's findings. Other researchers ${ }^{(48)}$ identified significant correlations between low fruit and vegetable intake and irregular breakfast habits among 11-15-year-old children. Older female participants were shown to be at an especially higher risk of low fruit and vegetable intake ${ }^{(48)}$. In another recent publication, it was reported among Saudi adolescents that healthy behaviours, clustered together, were significantly associated with physical activity, whereas unhealthy dietary habits tended to associate with higher screen time ${ }^{(49)}$; this goes partly along with our findings.

Additionally, the similarities between the reported prevalence of overweight/obesity in the present study and in the study conducted on male university students ${ }^{(19)}$ may be related to one of the major environmental causes of obesity - that is, changes in diet, in terms of quantity and quality, a diet that has become more 'Westernized'(50) brought about by international fast-food chains. Most Saudi students $(63.3 \%)^{(19)}$ eat irregular meals, whereas $64.6 \%$ of Lebanese ${ }^{(51)}$ and $81.6 \%$ of Chinese ${ }^{(52)}$ male university students take regular meals. The eating habits of the KSA youth population need to be improved using educational programmes aimed at promoting healthy eating habits. The modernization and affluence in KSA over the last three decades are thought to have contributed largely to the rise in the obesity epidemic. Following this, strategies to prevent obesity among female university populations in KSA should include encouragement of eating behaviour modification such as reduced intake of high-fat foods and increased consumption of fruits and vegetables. Further, national nutritional guidelines and campaigns should be developed further and integrated into all educational curriculums. Also healthier food choices should be available for university students during study days.

The influence of sisters on the study participants' fresh fruit intake and sweets consumption might be related to the cultural and environmental effects on meal consumption; that is, of sharing the meals with family. The family meals were found to be of great importance in influencing adolescent food choices ${ }^{(53)}$. A recently published study in the USA ${ }^{(54)}$ found that frequently shared meals in young adulthood were associated with a greater intake of fruit among males and females, and with a higher intake of vegetables, milk products and some key nutrients among females. Furthermore, fruits and sweets might be consumed at social events and family gathering moments ${ }^{(55)}$, where sisters usually gather. A recent study showed that women more frequently make healthier dietary food choices and are more likely to consume fruits as snacks ${ }^{(56)}$ in comparison to men. Another possible reason for the influence of sisters, either as models for eating behaviours or as social peers, might be the nature of KSA culture, where women usually dine separately from men, especially in large gatherings ${ }^{(57)}$. Thus, the most effective way to prevent an unhealthy intake of sweets and encourage healthy fruit consumption would be to increase healthy food campaigns targeting the family as a whole. As a consequence of such action, the obesity-promoting eating habits might be targeted, leading to a possibly healthier female population in the KSA.

Furthermore, the present study introduced an interesting aspect: an increased number of sisters significantly correlated with developing either overweight/obesity or underweight. This contradictory finding might be related to the earlier mentioned cultural structure of KSA society ${ }^{(57)}$, which means an increased socialization of sisters in the house environment. While some studies ${ }^{(58)}$ found that a larger number of siblings decreased the odds for overweight ( $P$ for trend $<0 \cdot 001$ ), other studies ${ }^{(59)}$ tried to research the potential mechanisms explaining correlated BMI outcomes in a biologically related social network. In that study, the researchers found that time-constant factors such as genetic heritability and habits formed during childhood explain some of the overall correlation in sibling $\mathrm{BMI}^{(59)}$. Further, they found that factors that change over time - for example, social norms or environmental factors like opportunities for exercise - significantly impact the overall correlation in BMI only for adolescent siblings, suggesting that the influence of the social network on correlations in BMI is facilitated by sharing the same household ${ }^{(59)}$.

Although the present study offers insight into the current nutritional and weight status in a healthy, highly educated sample, a number of limitations exist. Generalizability is limited because of the selectivity of the study setting. On the other hand, strengths of the present epidemiological study could be that the participants were considered representative of the studied population, the used protocol is a reproducible and validated questionnaire, and the study's procedures were highly standardized. Furthermore, the questionnaire is comparable to other self-reporting instruments on the whole ${ }^{(39)}$. The study results should, therefore, be generalizable to other female university students, not only in the KSA but also in other Arab countries. Another potential shortcoming of the present study might be the rigour with which its results can be applied to expectations 
of physical activity behaviour of the entire Saudi population because the study participation was limited to female university students. In addition, these students lived at a high altitude (2000-2300 m above sea level), which might make this sample population environmentally different compared with the rest of the Saudi population. Further, we assessed only the frequency of eating without accounting for portion size, which might have given additional information about the dietary habits of this population. For example, in a Swedish study, obesity was reported to be significantly associated with larger self-reported portion sizes at main meals ${ }^{(60)}$.

\section{Conclusions}

The coexistence of underweight and overweight/obesity in an affluent society like KSA should be a target for further research, especially among the female population. The results of the present study could be useful to healthcare providers and policy makers to prevent many health conditions related to underweight such as tuberculosis, osteoporosis and infectious diseases, as well as noncommunicable diseases related to overweight/obesity. The study can be an evidence-based background for planning and implementation of interventions targeting improvement of highly educated populations' nutritional habits. Furthermore, we suggest that strategies to prevent the prevailing obesity in KSA should involve the family to encourage sustainable changes in lifestyle patterns such as increased physical activity and healthier nutritional choices and habits.

\section{Acknowledgements}

Acknowledgements: The authors would like to thank the PRO-CARE (Patient Reported Outcomes - Clinical Assessment, Research and Education) research group at Kristianstad University, Sweden, for its contribution in revising the manuscript critically. They also thank the Research Council at Kristianstad University for its support. Special thanks are extended to the following people for their critical role in acquisition of data: Dr Rania Mahmoud Abdel Ghani, Maternal and Newborn Health Nursing, Faculty of Nursing, Cairo University, Egypt; Mrs Jipi Varghese, Department of Medical Surgical Nursing, College of Nursing, King Khalid University, Saudi Arabia; and Mrs Iman Al-Hissi, Pediatric Nursing, Faculty of Nursing, Cairo University, Egypt. Financial support: The whole project was funded by the Research Council at Kristianstad University (grant number 059/09). The Research Council at Kristianstad University had no role in the design, analysis or writing of this article. Conflict of interest: None. Authorship: A.K. had the main responsibility for the data collection, analysis and interpretation as well as drafting the manuscript. A.W. contributed to the design of the study, performing the statistical analysis and drafting the manuscript. V.B.'s contribution included study design and drafting of the manuscript. Ö.E. participated in performing the statistical analysis and helped to draft the manuscript. H.M.A.-H. contributed to the conception, design and coordination of the study, and helped to draft the manuscript. All authors read and approved the final manuscript. Ethics of buman subject participation: The study was reviewed and approved by the Ethical Committee at King Khalid University, Abha, KSA (7/1078).

\section{References}

1. World Health Organization (2013) World Health Statistics, A Wealth of Information on Global Public Health. Geneva: WHO.

2. Popkin BM, Adair LS \& Ng SW (2012) Global nutrition transition and the pandemic of obesity in developing countries. Nutr Rev 70, 3-21.

3. James WPT, Jackson-Leach R, Ni Mhurchu C et al. (2004) Overweight and obesity (high body mass index). In Comparative Quantification of Health Risks: Global and Regional Burden of Disease Attributable to Selected Major Risk Factors, vol. 1, pp. 497-596 [M Ezzati, AD Lopez, A Rodgers et al., editors]. Geneva: WHO.

4. Struber J (2004) Considering physical inactivity in relation to obesity. Internet J Allied Health Sci Pract 2, 1-13.

5. ten Hacken NH (2009) Physical inactivity and obesity: relation to asthma and chronic obstructive pulmonary disease? Proc Am Thorac Soc 6, 663-667.

6. Pedersen BK (2009) The diseasome of physical inactivity and the role of myokines in muscle-fat cross talk. J Physiol 587, 5559-5568.

7. Lee I-M, Shiroma EJ, Lobelo F et al. (2012) Effect of physical inactivity on major non-communicable diseases worldwide: an analysis of burden of disease and life expectancy. Lancet 380, 219-229.

8. El Mouzan MI, Foster PJ, Al Herbish AS et al. (2010) Prevalence of overweight and obesity in Saudi children and adolescents. Ann Saudi Med 30, 203-208.

9. Al-Nozha MM, Al-Mazrou YY, Al-Maatouq MA et al. (2005) Obesity in Saudi Arabia. Saudi Med J 26, 824-829.

10. Khalaf A, Berggren V, Al-Hazzaa H et al. (2011) Undernutrition risk, overweight/obesity, and nutritional care in relation to undernutrition risk among inpatients in southwestern Saudi Arabia. A hospital-based point prevalence study. J Nutr Disord Ther 1, 104, doi:10.4172/jndt.1000104.

11. Al-Nuaim AA, Al-Nakeeb Y, Lyons M et al. (2012) The prevalence of physical activity and sedentary behaviours relative to obesity among adolescents from Al-Ahsa, Saudi Arabia: rural versus urban variations. J Nutr Metab 2012, 417589.

12. Madani KA (2000) Obesity in Saudi Arabia. Babrain Med Bull 22, 113-118.

13. Aboul-Enein FH (2002) Personal contemporary observations of nursing care in Saudi Arabia. Int J Nurs Pract 8 , 228-230.

14. bin Zaal AA, Musaiger AO \& D'Souza R (2009) Dietary habits associated with obesity among adolescents in Dubai, United Arab Emirates. Nutr Hosp 24, 437-444.

15. Khalaf A (2014) Nutrition, weight status and physical activity in Saudi Arabia - with special focus on women. PhD Thesis, Karolinska Institute.

16. El-Hazmi MA \& Warsy AS (2002) A comparative study of prevalence of overweight and obesity in children in different provinces of Saudi Arabia. J Trop Pediatr 48, 172-177. 
17. Al-Hazzaa HM (2010) Physical inactivity in Saudi Arabia. An underserved public health issue. Saudi Med J 31, $1278-1279$.

18. Sisson SB \& Katzmarzyk PT (2008) International prevalence of physical activity in youth and adults. Obes Rev 9, 606-614.

19. Al-Rethaiaa AS, Fahmy AE \& Al-Shwaiyat NM (2010) Obesity and eating habits among college students in Saudi Arabia: a cross sectional study. Nutr J 9, 39.

20. Collison KS, Zaidi MZ, Subhani SN et al. (2010) Sugarsweetened carbonated beverage consumption correlates with BMI, waist circumference, and poor dietary choices in school children. BMC Public Health 10, 234.

21. Rampersaud GC, Pereira MA, Girard BL et al. (2005) Breakfast habits, nutritional status, body weight, and academic performance in children and adolescents. J Am Diet Assoc 105, 743-760.

22. Al-Hazzaa HM, Abahussain NA, Al-Sobayel HI et al. (2012) Lifestyle factors associated with overweight and obesity among Saudi adolescents. BMC Public Health 12, 354.

23. Abalkhail B \& Shawky S (2002) Prevalence of daily breakfast intake, iron deficiency anaemia and awareness of being anaemic among Saudi school students. Int J Food Sci Nutr 53, 519-528.

24. AlQuaiz AM \& Tayel SA (2009) Barriers to a healthy lifestyle among patients attending primary care clinics at a university hospital in Riyadh. Ann Saudi Med 29, 30-35.

25. Ha D, Feskens E, Deurenberg P et al. (2011) Nationwide shifts in the double burden of overweight and underweight in Vietnamese adults in 2000 and 2005: two national nutrition surveys. BMC Public Health 11, 62.

26. World Health Organization (2006) Global Database on Body Mass Index. http://apps.who.int/bmi/index.jsp (accessed September 2013).

27. Mendez MA, Monteiro CA \& Popkin BM (2005) Overweight exceeds underweight among women in most developing countries. Am J Clin Nutr 81, 714-721.

28. Ritz BW \& Gardner EM (2006) Malnutrition and energy restriction differentially affect viral immunity. $J$ Nutr 136 1141-1144.

29. Jones JM, Bennett S, Olmsted MP et al. (2001) Disordered eating attitudes and behaviours in teenaged girls: a schoolbased study. CMAJ 165, 547-552.

30. Strelan P, Mehaffey SJ \& Tiggemann M (2003) Brief report: self-objectification and esteem in young women: the mediating role of reasons for exercise. Sex Roles 48, 89-95.

31. Musaiger AO, Shahbeek NE \& Al-Mannai M (2004) The role of social factors and weight status in ideal body-shape preferences as perceived by Arab women. J Biosoc Sci 36, 699-707.

32. Keski-Rahkonen A, Kaprio J, Rissanen A et al. (2003) Breakfast skipping and health-compromising behaviors in adolescents and adults. Eur J Clin Nutr 57, 842-853.

33. Choi O, Cho YG, Kang JH et al. (2013) Weight control attempts in underweight Korean adults: Korea National Health and Nutrition Examination Survey, 2007-2010. Korean J Fam Med 34, 393-402.

34. Rasheed P (1998) Perception of body weight and selfreported eating and exercise behaviour among obese and non-obese women in Saudi Arabia. Public Health 112, 409-414.

35. Al-Hazzaa H, Almuzaini K \& Al-Refaee S (2002) Assessment of Body Composition on College-Age Students: Comparison of Air Displacement Plythesmography, BIA, and Skinfolds. Riyadh: Educational Research Center, King Saud University Press.

36. Khalaf A, Ekblom Ö, Kowalski J et al. (2013) Female university students' physical activity levels and associated factors - a cross-sectional study in southwestern Saudi Arabia. Int J Environ Res Public Health 10, 3502-3517.
37. Al-Hazzaa HM, Al-Sobayel HI \& Musaiger AO (2011) Convergent validity of the Arab Teens Lifestyle Study (ATLS) physical activity questionnaire. Int J Environ Res Public Health 8, 3810-3820.

38. Al-Hazzaa HM \& Musaiger AO (2011) Arab Teens Lifestyle Study (ATLS): objectives, design, methodology and implications. Diabetes Metab Syndr Obes 4, 417-426.

39. Ainsworth BE, Haskell WL, Herrmann SD et al. (2011) 2011 compendium of physical activities: a second update of codes and MET values. Med Sci Sports Exerc 43, 1575-1581.

40. World Health Organization (2003) Obesity and Overweight. Geneva: WHO.

41. Altman DG (editor) (1991) Practical Statistics for Medical Research. London: Chapman and Hall, CRC Press.

42. Al-Mukhtar RA \& Musaiger A (2000) Obesity in female students in the United Arab Emirates University. Babrain Med Bull 22, 136-137.

43. El-Mouzan MI, Al-Salloum AA, Al-Herbish AS et al. (2010) Effects of education of the head of the household on the prevalence of malnutrition in children. Saudi Med J 31, 304-307.

44. El Mouzan MI, Al Herbish AS, Al Salloum AA et al. (2012) Regional variation in prevalence of overweight and obesity in Saudi children and adolescents. Saudi J Gastroenterol 18, 129-132.

45. Podewils L, Holtz T, Riekstina V et al. (2011) Impact of malnutrition on clinical presentation, clinical course, and mortality in MDR-TB patients. Epidemiol Infect 139, $113-120$.

46. Lindstrand A, Bergström S, Rosling H et al. (2006) Global Health: An Introductory Textbook. Lund: Studentlitteratur.

47. Tanaka S, Kuroda T, Saito M et al. (2013) Overweight/ obesity and underweight are both risk factors for osteoporotic fractures at different sites in Japanese postmenopausal women. Osteoporos Int 24, 69-76.

48. Lazzeri G, Pammolli A, Azzolini E et al. (2013) Association between fruits and vegetables intake and frequency of breakfast and snacks consumption: a cross-sectional study. Nutr J 12, 123.

49. Al-Hazzaa H, Al-Sobayel H, Abahussain N et al. (2014) Association of dietary habits with levels of physical activity and screen time among adolescents living in Saudi Arabia. J Hum Nutr Diet 27, Suppl. 2, 204-213.

50. Gasbarrini A \& Piscaglia AC (2005) A natural diet versus modern Western Diets? A new approach to prevent 'wellbeing syndromes'. Dig Dis Sci 50, 1-6.

51. Yahia N, Achkar A, Abdallah A et al. (2008) Eating habits and obesity among Lebanese university students. Nutr $J$ 7, 32.

52. Sakamaki R, Toyama K, Amamoto R et al. (2005) Nutritional knowledge, food habits and health attitude of Chinese university students - a cross sectional study. Nutr J $\mathbf{4}, 4$.

53. Neumark-Sztainer D, Larson NI, Fulkerson JA et al. (2010) Family meals and adolescents: what have we learned from Project EAT (Eating Among Teens)? Public Health Nutr 13, $1113-1121$.

54. Larson N, Fulkerson J, Story M et al. (2013) Shared meals among young adults are associated with better diet quality and predicted by family meal patterns during adolescence. Public Health Nutr 16, 883-893.

55. Pedersen S, Grønhøj A \& Bech-Larsen T (2012) Family members' roles in healthy-eating socialization based on a healthy-eating intervention. Young Consumers: Insight and Ideas for Responsible Marketers 13, 208-223.

56. Hartmann C, Siegrist M \& van der Horst K (2013) Snack frequency: associations with healthy and unhealthy food choices. Public Health Nutr 16, 1487-1496.

57. Mobaraki AH \& Söderfeldt B (2010) Gender inequity in Saudi Arabia and its role in public health. East Mediterr Health J 16, 113-118. 
58. Ochiai H, Shirasawa T, Ohtsu T et al. (2012) Number of siblings, birth order, and childhood overweight: a population-based cross-sectional study in Japan. BMC Public Health 12, 766.

59. Brown HW \& Roberts J (2012) Exploring the factors contributing to sibling correlations in BMI: a study using the
Panel Study of Income Dynamics. Obesity (Silver Spring) 20, 978-984.

60. Berg C, Lappas G, Wolk A et al. (2009) Eating patterns and portion size associated with obesity in a Swedish population. Appetite 52, 21-26. 